\title{
Evaluación del estado de elementos estructurales del Mercado de Le- gazpi: Ataque por sulfatos al hormigón y corrosión de las armaduras
}

\section{Evaluation of the state of structural elements of the Legazpi Market: Attack by sulphates on concrete and corrosion of reinforcements}

\begin{abstract}
Servando Chinchón-Payá( $^{(*)}$, Julio Torres ${ }^{(* *)}$, Nuria Rebolledo ${ }^{(* *)}, J^{*}$ avier Sánchez ${ }^{(* * *)}$
RESUMEN

El presente trabajo describe la metodología empleada para el estudio del estado del hormigón de zapatas, soportes, así como del terreno en varias zonas del edificio del Mercado de Legazpi en Madrid (España). Se han analizado los pilares, los arranques de pilar, las zapatas y los terrenos donde estos últimos están enterrados. Se ha realizado una inspección visual y una caracterización por ultrasonidos, lo cual ha permitido la selección de muestras para el posterior análisis en laboratorio mediante difracción de rayos X y microscopía electrónica de barrido, así como cuantificación de cloruros y sulfatos. Se han encontrado afecciones de corrosión de las armaduras en zonas puntuales, que son debidas tanto al anterior uso de la estructura como al envejecimiento de la misma. También existe una importante cantidad de sulfatos en los terrenos, de origen antrópico, que explicarían la formación de productos de ataque sulfático en las zapatas. El ataque por sulfatos, aunque presente, no parece suponer una merma importante en la integridad de la estructura.
\end{abstract}

Palabras clave: Durabilidad; hormigón; corrosión; ataque externo por sulfatos; Legazpi

\section{ABSTRACT}

The work describes the methodology used to study the concrete of pillars, supports and foundations of several zones of the Mercado de Legazpi in Madrid (Spain). The soils where the latter are buried have also been analyzed. A visual inspection together with an Ultrasonic characterization has allowed the selection of samples for subsequent laboratory analysis by X-Ray Diffraction and Scanning Electron Microscopy, as well as quantification of chlorides and sulphates. Corrosion of reinforcements have been found in specific areas, which are due both to the previous use of the structure and its aging. There is also a significant amount of sulfates in the soil, of anthropogenic origin, which would explain the formation of sulphate attack products in the foundations. However, this attack does not seem to be a significant decrease in the integrity of the structure.

Keywords: Durability; concrete; corrosion; external sulphate attack; Legazpi

(*) Dr. CC. Químicas. Investigador contratado. IETcc, Madrid (España).

(**) Dr. Ing. Materiales. Técnico superior. IETcc, Madrid (España).

(**) Ing. Química. Técnico superior. IETcc, Madrid (España).

(***) Dr. Ing. Químico. Investigador Ttular. IETcc, Madrid (España).

Persona de contacto/Corresponding author: servando@ietcc.csic.es (S. Chinchón-Payá)

ORCID: https://orcid.org/oooo-00o2-2649-7188 (S. Chinchón-Payá); https://orcid.org/oooo-0002-1261-9199 (J. Torres); https://orcid.org/oooo-0001-7980-7971 (N. Rebolledo); https://orcid.org/oooo-0002-4334-0553 (J. Sánchez)

Cómo citar este artículo/Citation: Chinchón-Payá, Servando; Torres, Julio; Rebolledo, Nuria; Sánchez, Javier; (2021). Evaluación del estado de elementos estructurales del Mercado de Legazpi: Ataque por sulfatos al hormigón y corrosión de las armaduras. Informes de la Construcción, 73(561): e380. https://doi.org/10.3989/ic.76737

Copyright: (C) 2021 CSIC. Este es un artículo de acceso abierto distribuido bajo los términos de la licencia de uso y distribución Creative Commons Reconocimiento 4.0 Internacional (CC BY 4.0). 


\section{INTRODUCCIÓN}

El hormigón armado es el material de construcción más importante dado que une a la capacidad resistente del hormigón la propia de las armaduras de acero embebidas en el mismo. Sin embargo, tanto el hormigón como las armaduras se pueden degradar o deteriorar por la acción de agentes ambientales.

Por un lado, las armaduras se encuentran recubiertas por una finísima lámina de óxidos denominada capa pasiva (1-3). Dicha capa protege al acero del avance de la oxidación cuando las condiciones de $\mathrm{pH}$ del medio que lo rodea son lo suficientemente básicas, fenómeno conocido como "pasivación". El acero del hormigón armado está pasivado gracias a las condiciones básicas de los poros del cemento que lo rodea (4-6). Sin embargo, las condiciones de protección pueden desaparecer cuando el hormigón se carbonata o cuando los iones cloruro alcanzan la armadura en una concentración suficiente $(7,8)$.

Cuando las armaduras se corroen pierden sección provocando diversos efectos en las estructuras como el spalling del recubrimiento, pérdida de adherencia o fallo frágil de las propias armaduras, lo cual conduce a la pérdida de capacidad resistente de las mismas (9-11). Existen diversas técnicas y métodos para comprobar in-situ el estado de las armaduras (4, 12-18).

Por otra parte, el hormigón también se puede deteriorar y una de las afecciones que puede sufrir es el ataque por sulfatos. Los iones sulfatos pueden provenir del exterior cuando la estructura se encuentra en contacto con terrenos o aguas que contienen sales con alta concentración de sulfatos (19), o del mismo interior del hormigón cuando se han utilizado materiales de partida con altos contenidos en sulfatos, bien por el uso de un exceso de yeso como retardante del fraguado añadido durante la fabricación del cemento, bien por el uso de áridos contaminados con sulfuros de hierro (20), por yeso, $\mathrm{u}$ otros contaminantes (21).

Las reacciones que se producen son las siguientes:

- Formación de etringita secundaria (22) en la que los aluminatos hidratados reaccionan con los sulfatos de forma

$$
\begin{aligned}
& 6 \mathrm{Ca}^{2+}+\mathrm{Al}(\mathrm{OH})^{4-}+4 \mathrm{OH}^{-}+3 \mathrm{SO}_{4}^{2-}+26 \mathrm{H}_{2} \mathrm{O} \rightarrow \\
& \mathrm{Ca}_{6}\left(\mathrm{Al}(\mathrm{OH})_{6}\right) 2 \cdot\left(\mathrm{SO}_{4}\right)_{2} \cdot 26 \mathrm{H}_{2} \mathrm{O}
\end{aligned}
$$

- Formación de taumasita según (23), y donde se considera “ $\mathrm{Ca}_{3} \mathrm{Si}_{2} \mathrm{O}_{7} \cdot 3 \mathrm{H}_{2} \mathrm{O}$ " como composición característica del gel amorfo C-S-H.

$$
\begin{aligned}
& " \mathrm{Ca}_{3} \mathrm{Si}_{2} \mathrm{O}_{7} \cdot 3 \mathrm{H}_{2} \mathrm{O} "+2 \mathrm{CaSO}_{4} \cdot 2 \mathrm{H}_{2} \mathrm{O}+\mathrm{CaCO}_{3}+\mathrm{CO}_{2}+ \\
& 23 \mathrm{H}_{2} \mathrm{O} \rightarrow \mathrm{Ca}_{6}\left(\mathrm{Si}(\mathrm{OH})_{6}\right)_{2}\left(\mathrm{CO}_{3}\right)_{2}\left(\mathrm{SO}_{4}\right)_{2} \cdot 24 \mathrm{H}_{2} \mathrm{O}
\end{aligned}
$$

La etringita secundaria, llamada así para diferenciarla de la primaria formada en los primeros estadios de la hidratación del cemento, se forma al reaccionar los aluminatos cálcicos hidratados del cemento con los iones sulfatos. Se produce un aumento de volumen que crea tensiones internas y que puede derivar a una fisuración en la pasta (24).

La taumasita es un compuesto cristalino de parecida morfología a la etringita. También se forma por reacción de los sulfatos con componentes del cemento hidratado. Sin embargo, ya no se produce la reacción con los aluminatos cálcicos hidratados sino con los silicatos y los carbonatos presentes en el hormigón formando una sal compleja. La principal consecuencia de que se produzca esta reacción es la desintegración del gel CSH por reacción con los silicatos cálcicos que lo componen.

Dada la parecida morfología de taumasita y etringita, en el pasado se ha podido obviar la presencia de la primera pensando que se trataba de etringita. Es a partir de inicios de siglo cuando se toma conciencia de este compuesto por primera vez, viéndose el interés internacional materializado en un congreso monográfico (25) donde se enumeraron varios casos de estructuras reales afectadas, así como experiencias de laboratorio encaminadas a conocer este compuesto en mayor detalle.

En España no se han reportado muchas estructuras de hormigón que haya sufrido un ataque sulfático con formación de taumasita. En (26), los autores señalan un caso de formación en el hormigón de la presa de Mequinenza, construcción ubicada en terrenos con lignitos de alto contenido de sulfuros de hierro, la taumasita aparece en las canaletas del interior de las galerías por donde circula agua constantemente como consecuencia de un ataque externo por sulfatos. Otros casos de aparición de taumasita en España son (27) (Valle de los Caídos) donde se produce por incompatibilidad de los materiales usados, yeso; en (28) la aparición de taumasita se asume como la causa del fallo en un pavimento y en (29) se relaciona en un estudio sobre metodología de reparación de yesos históricos. La aparición de taumasita conlleva la pérdida de capacidad cohesiva del gel CSH y, en caso de que se forme en gran cantidad, puede llegar a convertir al hormigón en una masa que se desprende fácilmente y se desintegra con una leve presión manual (26).

El objetivo principal del presente trabajo es conocer el estado actual de la estructura del antiguo mercado de Legazpi después de 83 años desde su construcción. Se presenta una metodología de trabajo que incluye la inspección visual del 100\% de los elementos de la estructura, la caracterización in-situ a través de la velocidad de ultrasonidos, la resistividad y las medidas de potencial de corrosión y velocidad de corrosión, y la obtención de muestras para evaluar posibles procesos de deterioro.

\section{METODOLOGÍA}

El mercado de Legazpi, antiguo mercado central de frutas y verduras, está incluido en el Catálogo de edificios Protegidos del Plan General vigente en el Nivel 2, grado Estructural, fue diseñado a principios de siglo XX por un equipo referente en nuestro país en aquellos años, como son, los arquitectos Luís Bellido González y Francisco Javier Ferrero Llusía y el ingeniero Alfonso Peña Boeuf. La construcción, situada en la plaza de Legazpi en el madrileño distrito de Arganzuela, tardó en acabarse casi una década, inaugurándose en 1935 (aunque el periodo de construcción fue de 30 meses, existió un parón de 18 meses entre ellos por cuestiones administrativas, que extendió el tiempo a un total de 48 meses hasta su inauguración). El edificio de dos plantas ocupa una extensión de $47400 \mathrm{~m}^{2}$ construidos, siendo un tercio de ella en planta baja $\left(17500 \mathrm{~m}^{2}\right)$. El edificio cuenta con accesos para vehículos y carruajes desde un gran patio central de unos $9400 \mathrm{~m}^{2}$. En esta extensión tenían cabida más de 400 puestos de distinto tamaño, almacenes, distintos servicios del mercado y un bar. 
El edificio consta en total de 6 crujías en ambas plantas, de hormigón visto, con una distribución reticulada de pilares de 6x6x6 metros (la separación entre soportes es de 6,5 en las dos direcciones, excepto el ancho de la crujía de fachada que es de $4,5 \mathrm{~m}$ ), con viguetas transversales a la nave de crujías laterales, distribuidas uniformemente y viguetas longitudinales cargadas sobre vigas transversales en las zonas centrales específico para el paso de vehículos. Las fachadas de fábrica de ladrillo limitadas por los elementos estructurales de hormigón visto, en forma modular con ventanales metálicos que se extienden por todo el edificio para facilitar la iluminación natural en su interior. En su segunda planta la cubierta se compone de losas con hasta 6 metros de vuelo que permiten tener protegido el interior.

Se trata de un edificio sobre zapatas aisladas que apoyan en un firme situado a una profundidad variable. Las zapatas tienen forma troncopiramidal y un canto máximo de $60 \mathrm{~cm}$ y de ellas arrancan unas basas hasta la cota de solera, desde donde arrancan los pilares de planta baja. El espacio situado entre la cota del firme en que apoyan las zapatas y el apoyo de la solera es un relleno antrópico realizado después de la construcción de las zapatas y las basas.

En 1983 finalizó su actividad y explotación como mercado, coincidiendo con la puesta en marcha de "MercaMadrid". En los últimos años ha tenido diferentes usos, pero siempre en zonas muy limitadas del edificio como parking de vehículos del SAMUR, almacén, objetos perdidos, etc...

Entre 2008 y 2011 se realizó un proyecto para rehabilitar el edificio que finalmenteno se llevó a cabo. En 2015 se ha retomado la iniciativa realizándose una modificación del proyecto y en la actualidad sigue su consecución. En el proyecto se incluye la reparación de los daños existentes en la estructura del edificio, así como la rehabilitación para albergar oficinas municipales y servicios para el vecindario. Asimismo, se contempla la eliminación de los terrenos antrópicos por otros sin contenido en sulfatos.

\subsection{Ensayos no destructivos in-situ y toma de muestras.}

Una parte importante del estudio del estado del hormigón de la estructura se realiza mediante métodos no destructivos in-situ sobre los elementos de la misma. Para ello se utilizó una inspección visual, técnicas de ultrasonidos y electroquímicas (resistividad, potencial de corrosión y velocidad de corrosión).
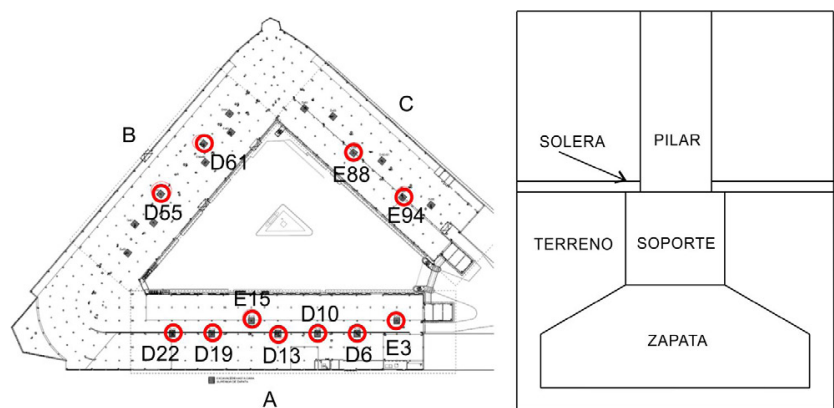

Figura 1.- Planta del mercado con las zonas de muestreo (izquierda) (Fuente: FERROVIAL); y esquema del sistema constructivo (derecha).
Las datos y observaciones realizadas han servido para conocer el estado general de la estructura e identificar las zonas que mayor degradación presentan. Gracias a ello se extrajeron un conjunto de muestras para analizarlas en el laboratorio.

Se analizó el hormigón de ciertos elementos en las tres diferentes zonas donde se asienta la estructura (ver Figura 1 izquierda), concretamente se tomaron muestras de hormigón de las zapatas, del arranque de pilar y de alguno de los pilares. Los pilares analizados se muestran en la Figura 1 izquierda. También se recogieron muestras del terreno circundante de cada uno de los pilares analizados. Se tomaron más muestras de la zona A porque en estudios anteriores se observó que los elementos de esta zona presentaban mayores signos de degradación. Esta zona fue la primera en abrirse y se tuvo acceso a una mayor cantidad de arranques de pilar y zapatas, mientras que las zonas $\mathrm{B}$ y C, solo se desenterraron algunas de las zapatas, de donde se sacó muestra.

En la Figura 1 derecha se muestra de forma esquemática los elementos estructurales analizados en este trabajo. Por debajo del nivel del suelo se encuentra el arranque de pilar y la zapata. En el caso de los arranques de pilar hay que indicar que no se consideró necesario realizar tantos análisis como en el caso de los demás elementos (terrenos, zapatas y pilares) dado que el estado del hormigón es muy bueno, sensiblemente mejor que el del resto de elementos. Durante las visitas al mercado se ha podido comprobar este hecho in situ. Corrobora este hecho la dificultad que se tuvo en recoger muestras de los mismos y los análisis de medidas de velocidad de ultrasonidos ya indicados.

\subsection{Estudios en laboratorio}

Para profundizar en el estudio del estado de la microestructura del hormigón se tomaron un gran número de muestras y se analizaron mediante un conjunto diverso de técnicas instrumentales tales como:

- Cuantificación $\left(\mathrm{SO}_{4}{ }^{2-}\right)$ y $\left(\mathrm{Cl}^{-}\right)$. Para la determinación de cloruros totales se hizo una valoración potenciométrica con electrodo selectivo. En el caso de la determinación de sulfatos la norma que se utilizó fue UNE 196-2.

- Microscopía óptica. Utilizada en las muestras sólidas para observar la apariencia general de las muestras, su nivel de degradación y la presencia y ubicación de cristales aciculares aparecidos como productos de reacción con sulfatos. Sirvió también para seleccionar partes que posteriormente se analizaron mediante microscopía electrónica y difracción de rayos $\mathrm{X}$.

- Microscopía electrónica de barrido (MEB-EDX). Microscopio JSM-540o de JEOL con capacidad de análisis mediante electrones secundarios y retro-dispersados. Utilizado para observar las muestras a grandes aumentos y analizar la composición elemental en los puntos deseados. Las muestras analizadas son pequeñas porciones sólidas de las extraídas.

- Difracción de rayos X (DRX). D8 Advance de BRUKER AXS sin monocromador y con anticátodo de $\mathrm{Cu}$. Utilizado para determinar la composición cristalina de la muestra y comprobar la posible aparición de productos de degradación. Para su análisis la muestra sólida hubo de ser pulverizada y secada previamente. Las condiciones de análisis fueron: barrido de $4^{\circ}$ a $60^{\circ} 2 \theta$ a una velocidad de $1^{\circ} / \mathrm{min}$. 


\section{RESULTADOS Y DISCUSIÓN}

\subsection{Estudios preliminares in situ}

Durante la visita se pudo observar que en general los pilares y forjados se encuentran en buen estado visual, aunque también se observó una zona del forjado en la que el recubrimiento se había perdido y la armadura inferior mostraba un alto grado de corrosión, esta zona está cercana a diferentes desagües y bajantes de la planta alta (Figura 2).

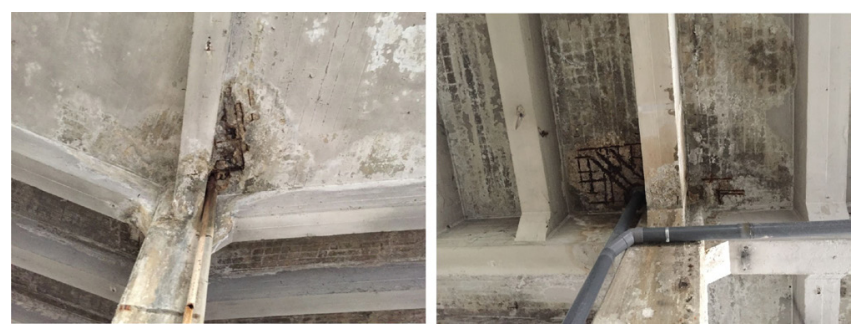

Figura 2.- Estado de parte inferior de forjados cercanos a desagües y bajantes de planta alta

Para estudiar el estado del hormigón de las basas (de los soportes) enterradas, se demolió la solera de la planta baja y se excavó en torno al soporte, llegando hasta la cara superior de la zapata en algunos casos. En estos pilares de nuevo se observaba de manera general buen estado visual tanto de las zapatas como de los pilares de la planta baja.

La medida de velocidad de ultrasonidos permite diferenciar distintas calidades de hormigón o delimitar zonas de degradación en los elementos de una misma estructura. En la inspección realizada se compararon zonas de distintos pilares de la estructura, centrándose en zona alta, entre 50 y $160 \mathrm{~cm}$ de forjado, zona baja, a $10 \mathrm{~cm}$ de forjado, zona de arranque de pilar, entre 20 y $100 \mathrm{~cm}$ bajo forjado y zona de las zapatas.

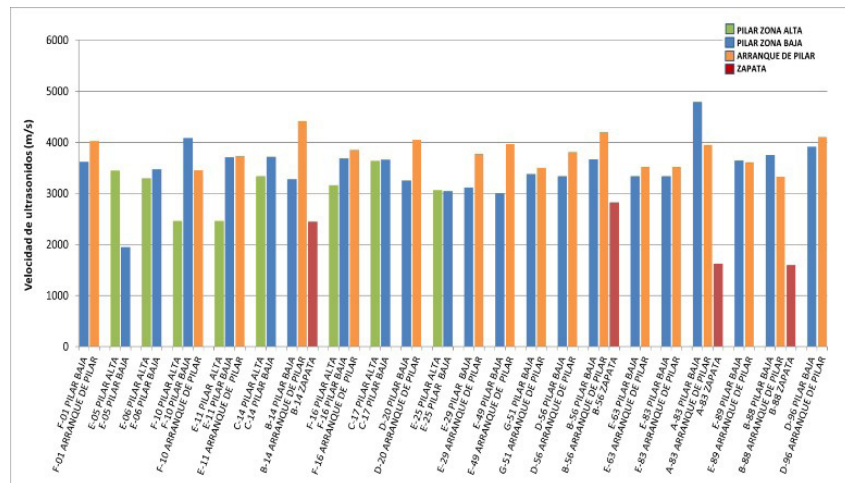

Figura 3.- Velocidad de ultrasonidos de elementos inspeccionados.

En la Figura 3 se observan los resultados de los ensayos de velocidad de ultrasonidos. El hormigón de los pilares de manera general tiene buena calidad, pero existen zonas de distintos elementos en las que su valor se encuentra por debajo del límite de $3000 \mathrm{~m} / \mathrm{s}$, considerándose (según las recomendaciones de (30)) de mala calidad o que puedan contener algún tipo de deterioro en su interior. Es de destacar que las zonas más afectadas son las zapatas, pero existen también zonas bajas de pilares que tienen mermada su calidad.

Estas medidas permitieron identificar diferentes calidades de hormigón. Las de calidad más baja se corresponden con la parte de la estructura situada en contacto directo con el terreno, pero no se tenía información sobre si existía, y en caso afirmativo, qué tipo de deterioro se estaba produciendo. En este sentido se obtuvieron muestras de terreno, de hormigón proveniente de las zapatas, del arranque del pilar y de la zona baja del pilar, a las cuales se les realizaron ensayos de contenido en sulfatos y cloruros, difracción de rayos X y microscopía electrónica de barrido.

\subsection{Estudios en laboratorio}

Se muestran a continuación los resultados del análisis de las muestras extraídas de la zona y la estructura analizadas mediante las distintas técnicas instrumentales.

\subsubsection{Análisis de los terrenos}

Se tomaron muestras del terreno en contacto con los pilares o elementos de la estructura que parecían más afectados. Los análisis que se realizaron fueron cuantificación de sulfatos y cloruros y difracción de rayos $\mathrm{X}$ para conocer los compuestos cristalinos presentes.

En la Tabla 1 se muestra la concentración de sulfatos y cloruros en las diferentes muestras analizadas.

Tabla 1.- Contenido de cloruros y sulfatos en las arenas que se encuentran en contacto con las zapatas. Color rojo para ataque fuerte

(Qa), color naranja para ataque medio $(\mathrm{Qb})$ y verde para ataque débil (Qc) según clasificación de la EHE para suelos agresivos.

\begin{tabular}{|c|c|c|c|}
\hline Zona & $\begin{array}{l}\text { Identificación } \\
\text { del tramo }\end{array}$ & $\begin{array}{c}\text { Cloruros to- } \\
\text { tales (\% peso } \\
\text { muestra seca) }\end{array}$ & $\begin{array}{c}\text { Sulfatos } \\
\text { (mg SO42-/kg } \\
\text { de arena seca) }\end{array}$ \\
\hline \multirow{7}{*}{$\mathbf{A}$} & E-O3 & 0,00 & 59040 \\
\hline & D-06 & 0,00 & 59811 \\
\hline & D-10 & 0,00 & 51225 \\
\hline & $\mathrm{D}-13$ & 0,00 & 33588 \\
\hline & E-15 & 0,00 & 34617 \\
\hline & D-19 & 0,00 & 38229 \\
\hline & $\mathrm{D}-22$ & 0,00 & 4176 \\
\hline \multirow{2}{*}{ B } & D-61 & 0,00 & 5478 \\
\hline & D-55 & 0,00 & - \\
\hline \multirow{2}{*}{$\mathbf{C}$} & E-88 & 0,00 & 2475 \\
\hline & E-94 & 0,00 & - \\
\hline
\end{tabular}

Se puede observar que los terrenos no contienen sales que aporten cloruros que disueltos en agua puedan provocar un ataque a las armaduras de la estructura.

Según la EHE-o8 (31) los límites considerados a partir de los cuales un terreno resulta agresivo se encuentran por encima de los $2000 \mathrm{mg}$ de sulfato/ $\mathrm{kg}$ de arena seca, por tanto, todas las arenas estudiadas se clasificarían en exposición Q, con el rango que se indica a continuación, encontrándose la mayoría de ellas situadas en el rango de ataque fuerte de sulfatos:

- Qa. Ataque débil. Concentraciones comprendidas entre 2.000 y $3.000 \mathrm{mg}$ de sulfato $/ \mathrm{kg}$ de arena seca (señaladas en la Tabla 1 en color verde).

- Qb. Ataque medio. Concentraciones comprendidas entre 3.000 y $12.000 \mathrm{mg}$ de sulfato/kg de arena seca (señaladas en la Tabla 1 en color naranja). 
- Qc. Ataque fuerte. Concentraciones superiores a $12.000 \mathrm{mg}$ de sulfato/kg de arena seca (señaladas en la Tabla 1 en color rojo).

Según los resultados obtenidos, los terrenos con el mayor contenido de sulfatos son los que se encuentran en la zona A, los cuales entran dentro de una clasificación $Q c$ pudiendo producir un ataque fuerte.

A continuación, se muestran los espectros de difracción de los terrenos analizados.

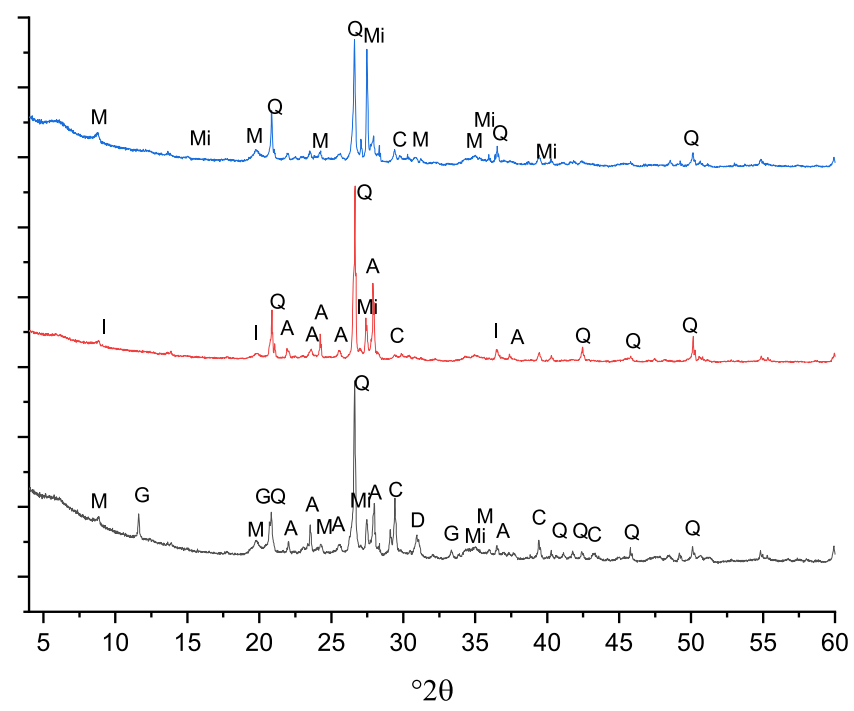

Figura 4.- DRX de las muestras de los terrenos extraídos de las inmediaciones en contacto con los pilares. Terreno D1o (negro), terreno D55 (rojo) y terreno E94 (azul). Q = Cuarzo, C = Calcita, M = Moscovita, $\mathrm{Mi}=$ Microclina, $\mathrm{A}=$ Albita, $\mathrm{I}=$ Illita y $\mathrm{G}=$ Yeso

La Figura 4 muestra los difractogramas de tres muestras de terrenos analizados. Se han seleccionado estos difractogramas dado que son representativos de las tres zonas del mercado (ver Figura 1). Según lo observado, la composición general del terreno es material silíceo-cálcico con gran cantidad de aluminosilicatos y feldespatos, sin embargo, la observación más importante que se puede hacer, en vista de estos resultados, es la presencia de gran cantidad de yeso (sulfato de calcio dihidratado) en los terrenos de una de las zonas del mercado, concretamente la zona A. La presencia de yeso en el terreno podría considerarse como la fuente de sulfatos detectados y mostrados en la Tabla 1.

\subsubsection{Análisis de las zapatas}

Las zapatas son elementos de hormigón enterrados en el suelo que presentan una gran porosidad y se desprenden con relativa facilidad sin necesidad de medios mecánicos.

La fotografía de la Figura 5 muestra la apariencia de la muestra de hormigón extraída de la zapata D55.

Se puede observar que el hormigón de las zapatas (se muestra como ejemplo la zapata D55, pero son todos de características similares) es un hormigón muy poroso y de poca cohesión. En parte de los poros se encuentran depósitos blanquecinos que pueden corresponder con los productos de ataque por sulfatos detectados.

Se muestran a continuación los resultados de la concentración de cloruros y sulfatos presentes en diferentes muestras de las zapatas de estos pilares.

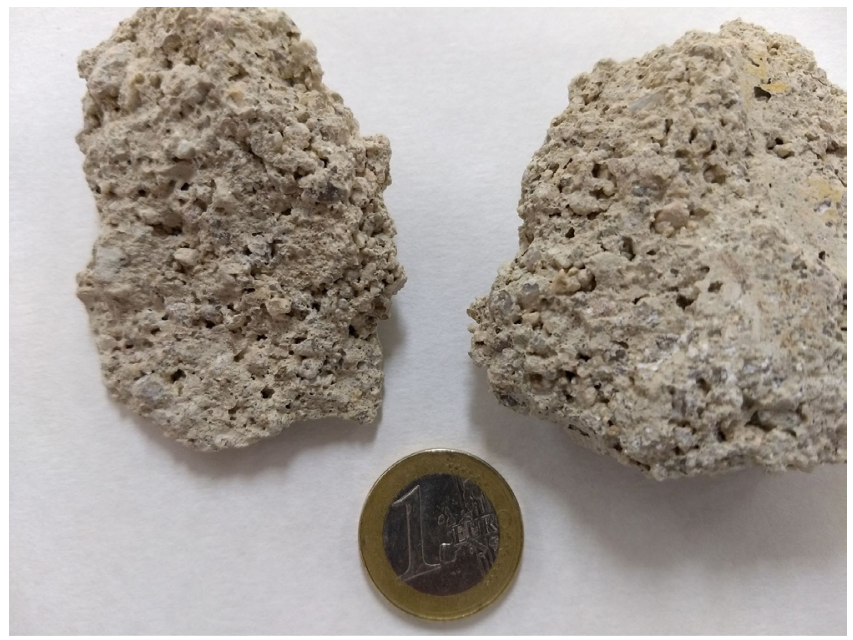

Figura 5.- Apariencia del hormigón de la zapata D55

Tabla 2.- Contenido de cloruros y sulfatos en muestras de hormigón de las zapatas.

\begin{tabular}{|c|c|c|c|c|c|}
\hline $\begin{array}{l}\stackrel{\mathfrak{Z}}{0} \\
\stackrel{0}{N}\end{array}$ & 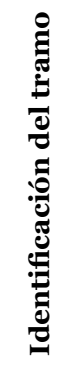 & 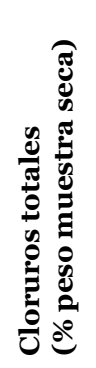 & 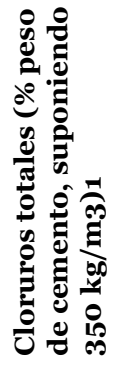 & 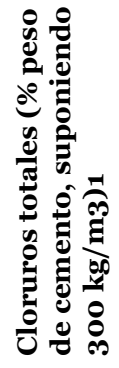 & 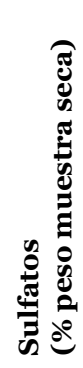 \\
\hline \multirow{7}{*}{ A } & E-03 & 0,03 & 0,25 & 0,21 & 2,18 \\
\hline & D-o6 & o & o & o & 1,20 \\
\hline & D-10 & 0,08 & 0,63 & 0,54 & 2,32 \\
\hline & $\mathrm{D}-13$ & 0,03 & 0,23 & 0,20 & 1,69 \\
\hline & E-15 & - & - & - & - \\
\hline & D-19 & o & o & o & 1,11 \\
\hline & D-22 & 0,03 & 0,20 & 0,17 & 1,60 \\
\hline \multirow{2}{*}{ B } & D-61 & - & - & - & - \\
\hline & D-55 & - & - & - & - \\
\hline \multirow{2}{*}{$\mathrm{C}$} & E-88 & 0 & 0 & 0 & 1,09 \\
\hline & E-94 & - & - & - & - \\
\hline
\end{tabular}

1 Los autores estiman contenidos de cemento 300 y $350 \mathrm{Kg} / \mathrm{m}^{3}$ de hormigón para poder obtener valores de cloruros expresados en peso de cemento (unidades normalizadas)

En la Tabla 2 se han calculado las concentraciones de cloruros en porcentaje de cemento que supondrían para un hormigón con un contenido de cemento entre 350 y $300 \mathrm{~kg} / \mathrm{m}^{3}$. Los autores desconocen la dosificación exacta, pero consideran esta cantidad de cemento mínima necesaria para este tipo de elementos estructurales. En 1935, al no existir instrucción que definiera la cantidad mínima de cemento, se usaban normas alemanas (DIN 1164 y DIN 1045 (1932)) que estimaban valores mínimos de cemento de entre $270-350 \mathrm{~kg}$ para la resistencia necesaria (32). Este umbral es necesario para disponer de valores de cloruros expresados en porcentaje en peso de cemento.

A su vez, en la Tabla 2 se han coloreado los valores de concentración que están por encima del límite de 0,6\%. Se puede observar que a excepción de algún valor de concentración 
cercano a 0,6 , las zapatas no presentan una concentración en cloruros que pueda suponen un ataque a las armaduras.

La concentración de sulfatos en las muestras analizadas es superior al establecido de 0,46 como propio de la naturaleza del hormigón, por lo que puede haber un aporte de dichos iones desde los terrenos circundantes.

Se muestran a continuación los espectros de DRX de las muestras de la zapata de pilar analizadas.

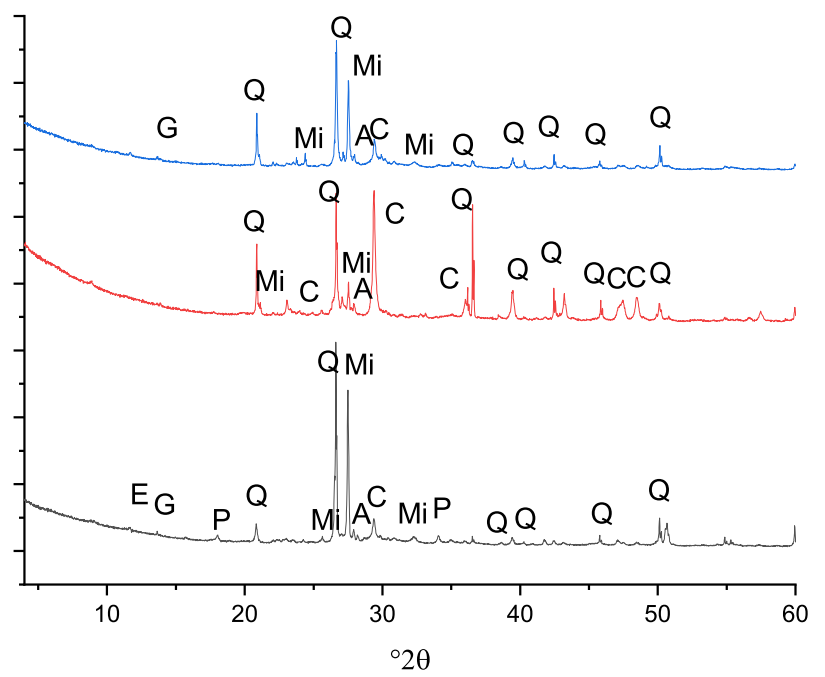

Figura 6.- DRX de las muestras de hormigón extraídas de las zapatas. Zapata D10 (negro), Zapata D55 (rojo) y zapata E94 (azul). $\mathrm{Q}=$ Cuarzo, $\mathrm{C}=$ Calcita, $\mathrm{Mi}=$ Microclina, $\mathrm{A}=$ Albita, $\mathrm{G}=$ Yeso, $\mathrm{P}=$

Portlandita, $\mathrm{E}=$ Etringita, $\mathrm{Mu}=$ Moscovita y Or = Ortoclasa.

En la Figura 6 se muestran los espectros de difracción de tres de las zapatas analizados. Las muestras corresponden a los mismos puntos analizados en los terrenos.

Se puede observar cierta cantidad de etringita y yeso en los análisis de la muestra la zapata D10. Aparece en esta muestra un poco de portlandita debido a una pequeña contaminación en la toma de muestras por presencia de material relativamente joven, de alguna de las actuaciones que se estaban llevando ya en la zona. En la zapata D55 se identifica calcita, cuarzo y aluminosilicatos en la forma de albita y microclima. Dada la cantidad de yeso observada en los terrenos de dicha zapata cabría esperar haber detectado algún producto de degradación por sulfatos en la matriz de cemento de esta zapata, pero sin embargo se puede observar que ni siquiera hay una mínima cantidad de etringita. En el espectro de la zapata E94 se observa yeso, que podría provenir como contaminación del suelo. No aparece, al igual que en la muestra anterior ninguna señal de etringita y por tanto de degradación.

Como se acaba de indicar, existen discrepancias en los análisis de los terrenos y las zapatas dado que algunas de las zapatas presentes en terrenos que dan altos contenidos de yeso, ni siquiera dan una mínima cantidad de etringita, es decir, de posibles productos de degradación. Por ello se han realizado análisis mediante microscopía óptica (MO) y microscopía electrónica de barrido (MEB). Los análisis de MO no se muestran, pero gracias a ello se han detectado ciertas zonas en alguna de las muestras que indican la posibilidad de tener productos de degradación de ataque sulfático. Los análisis de MEB se realizan para confirmar estas observaciones.
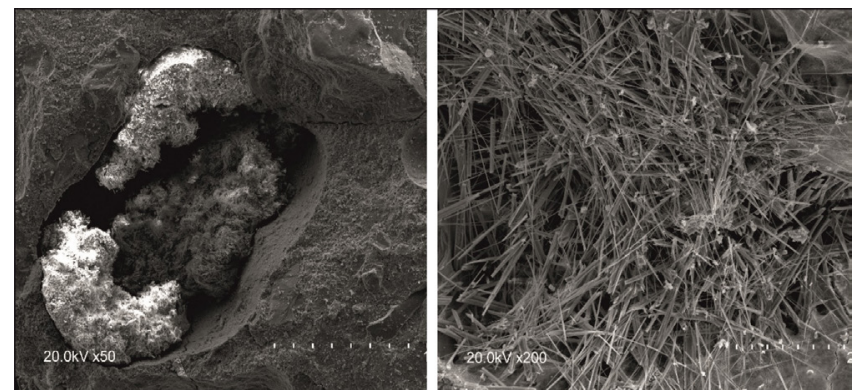

Figura 7.- Imágenes MEB de las muestras zapata D10 (izquierda) y D13 (derecha).

Se puede observar bastante cantidad de etringita secundaria en la muestra zapata D10 rellenando un macro poro, sin embargo, bastante cerca se encuentra otro poro completamente vacío de estos cristales. La superficie no se encuentra fisurada debido a expansiones por culpa de estos productos.

La imagen de la Figura 7 (derecha) muestra abundantes cristales aciculares de etringita en la superficie de la muestra zapata D13.

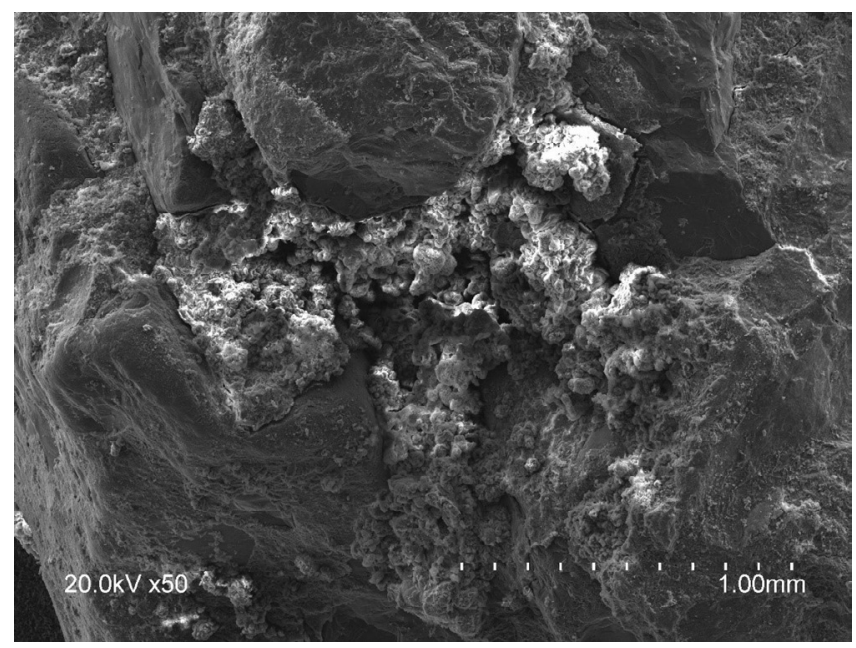

Figura 8.- Productos de degradación por ataque sulfático en la superficie de la muestra zapata D55.
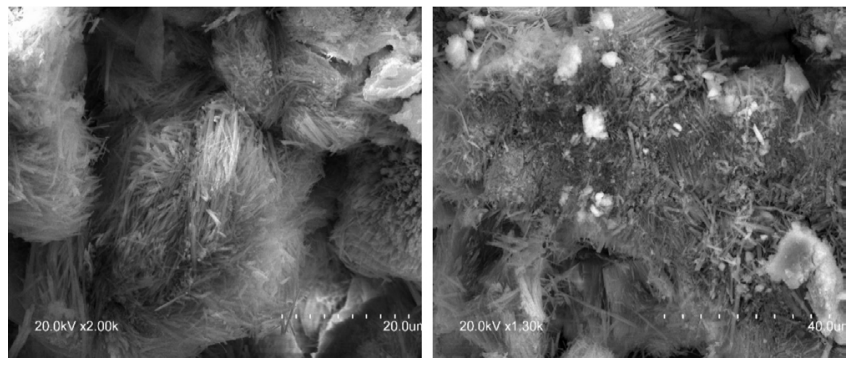

Figura 9.- Muestras de taumasita en zonas de la superficie de la muestra zapata D55 analizada.

De los análisis de microscopía de las muestras de las zapatas se puede observar que en varias de ellas aparecen productos de un ataque por sulfatos a los componentes de la pasta de cemento de los hormigones. Por ejemplo, la Figura 8 muestra la presencia de gran cantidad de productos de degradación por ataque sulfático en la superficie de la muestra de la zapata D55 a relativos pocos aumentos, detectables a simple vista. En la Figura 9 se muestra el aspecto de taumasita encontrada 


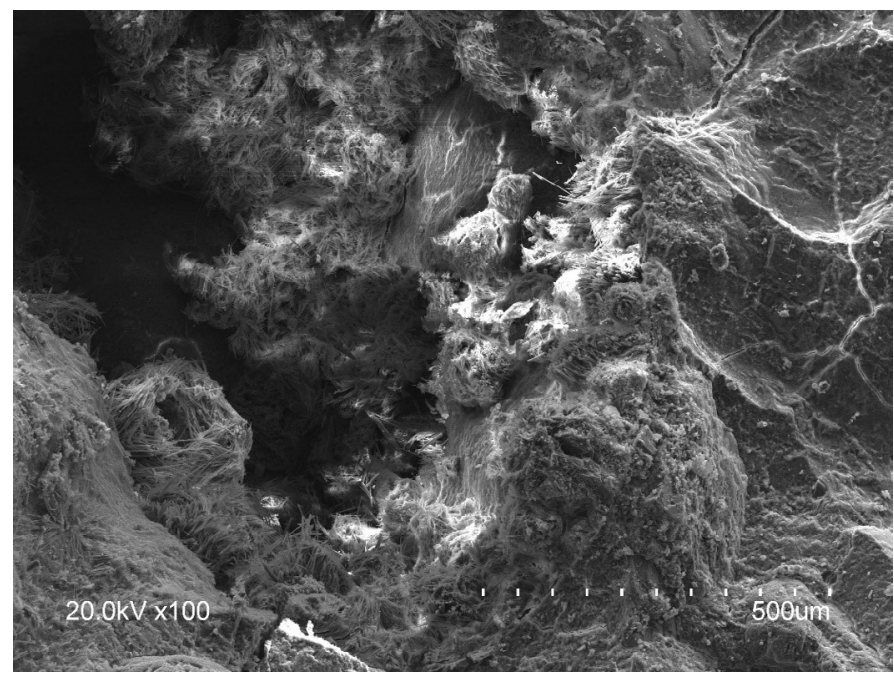

Figura 10.- Superficie de la muestra zapata E94.

en la muestra zapata D55. Este producto, también generado por una reacción de los sulfatos del terreno conlleva la descomposición del gel CSH.

La zapata D55 es la que más cantidad de productos de degradación presenta de todas las muestras analizadas. Sin embargo, los DRX no muestran la aparición de taumasita en concentración significativa y por tanto se considera su aparición como algo puntual y no representativo del conjunto del elemento.

La Figura 10 muestra el aspecto de la superficie de la muestra zapata E94, en la que se encuentra abundante cantidad de etringita. Se aprecian también fisuraciones producto de las expansiones provocadas al formarse este compuesto.

\subsubsection{Soportes o arranques de pilar}

Se muestran a continuación los análisis llevados a cabo de las muestras de hormigón extraídos de los arranques de pilar (Figura 11).

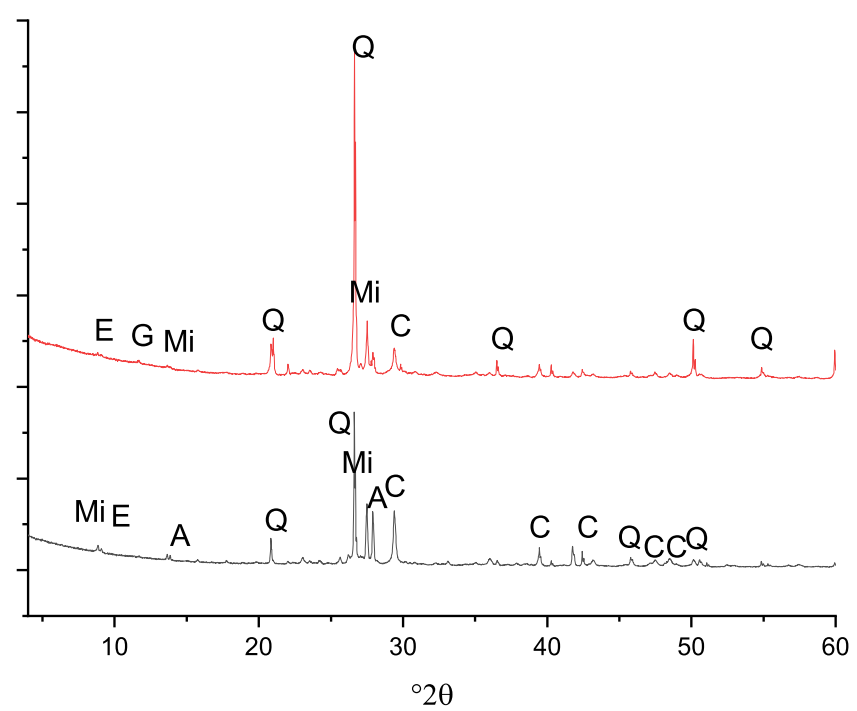

Figura 11.- DRX de muestras de los arranques de pilar D55 (negro) y D61 (rojo). $\mathrm{Q}=$ Cuarzo, $\mathrm{C}=$ Calcita, $\mathrm{Mi}=$ Microclina, $\mathrm{A}=$ Albita, $\mathrm{G}=$ Yeso y E = Etringita.
En los espectros se observa una composición del hormigón parecida a las de las zapatas pero con una menor concentración de feldespatos y micas, minerales propios de los terrenos. En ambos soportes aparece señal de cristales de etringita aunque esta es muy pequeña. Resulta llamativo el caso del soporte de pilar D55 en el que aparece etringita cuando en su zapata no se aprecia este compuesto. Es necesario indicar que la cantidad presente es mínima y no representativa de un taque severo por sulfatos.

\subsubsection{Pilares}

Los resultados de la cuantificación de cloruros y sulfatos en los pilares analizados son:

Tabla 3.- Contenido de cloruros y sulfatos en muestras de hormigón de los pilares.

\begin{tabular}{|c|c|c|c|c|c|}
\hline : & 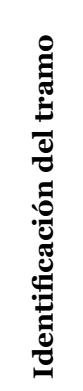 & 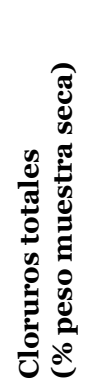 & 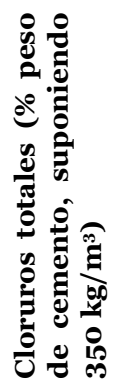 & 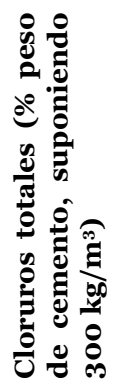 & 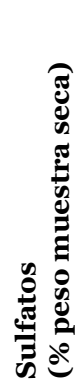 \\
\hline \multirow{7}{*}{$\mathbf{A}$} & E-03 & 0,48 & 3,71 & 3,18 & 2,35 \\
\hline & D-o6 & 0,39 & 2,98 & 2,55 & 2,34 \\
\hline & D-10 & 0,16 & 1,21 & 1,04 & 1,69 \\
\hline & D-13 & 0,30 & 2,33 & 1,99 & 1,97 \\
\hline & E-15 & 0,07 & 0,57 & 0,49 & 3,42 \\
\hline & D-19 & 0,05 & 0,40 & 0,34 & 1,75 \\
\hline & D-22 & 0,03 & 0,22 & 0,19 & 1,67 \\
\hline \multirow{2}{*}{ B } & D-61 & 0,04 & 0,27 & 0,23 & 1,24 \\
\hline & D-55 & 0,04 & 0,28 & 0,24 & 1,13 \\
\hline \multirow{2}{*}{ C } & E-88 & 0,05 & 0,36 & 0,31 & 1,03 \\
\hline & E-94 & - & - & - & - \\
\hline
\end{tabular}

La Tabla 3 muestra el contenido de cloruros y sulfatos para las muestras extraídas de la zona de los pilares. En este caso, vuelven a ser los elementos de la zona A, los que presentan una mayor concentración de cloruros, superando de nuevo lo indicado en la EHE-o8, que establece un valor de o,6\% de cloruros respecto del peso de cemento para la comprobación del Estado Límite en relación con la corrosión de las armaduras pasivas. Por tanto, se confirma que los cloruros pueden proceder de la actividad que se realizara en el mercado.

En el caso de los sulfatos, en todas las muestras analizadas se supera el valor de 0,46 , siendo muy acusado el caso de los pilares ubicados en la zona A. El resto de pilares tienen concentraciones de sulfatos parecidas al resto de muestras de hormigón, lo que conlleva a pensar que la mayor concentración de los iones sulfatos en los hormigones de los pilares de la zona A se deba también a causas del uso anterior de la estructura en esta zona.

Se muestran a continuación los espectros de difracción de los pilares analizados (Figura 12). 


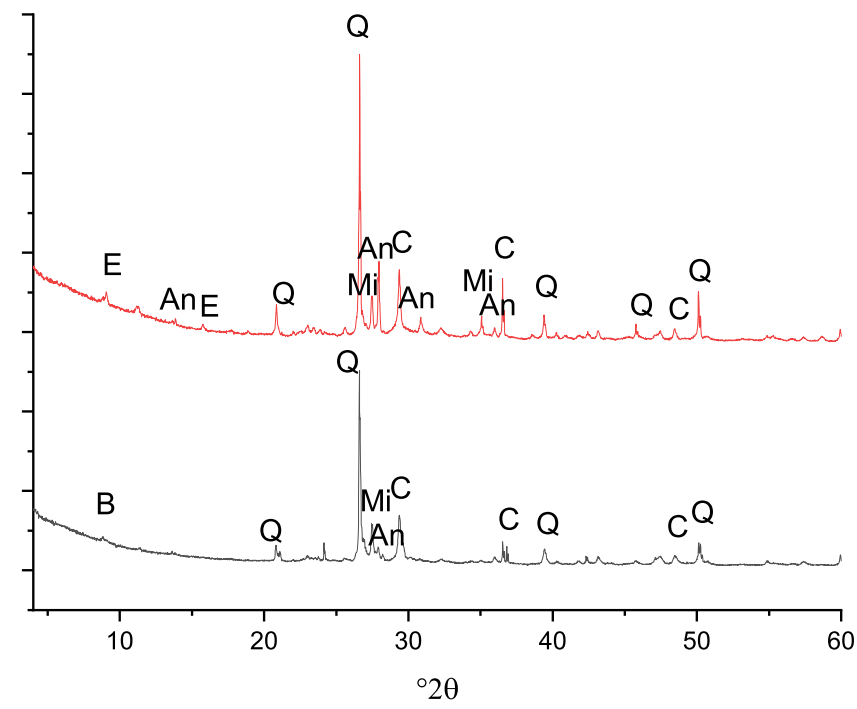

Figura 12.- DRX de las tres muestras de pilar D10 (rojo) y pilar D6 (negro). $\mathrm{Q}=$ Cuarzo, $\mathrm{C}=$ Calcita, $\mathrm{Mi}=$ Microclina, $\mathrm{An}=$ Anortita, $\mathrm{B}$ = Biotita $\mathrm{y} \mathrm{E}=$ Etringita

En el espectro correspondiente al pilar D1o se observa una cantidad importante de etringita, es el pilar que más cantidad de este producto presenta. En el pilar D6 no se detecta etringita, este pilar se encuentra en la misma zona que el pilar D10, en el que se acaba de ver que sí que se aprecia dicho compuesto posiblemente como producto de degradación.

La presencia de etringita únicamente en alguno de los pilares de una misma zona indica que no es un ataque por sulfatos generalizado a la estructura, sino que aparece en casos muy localizados en condiciones específicas (por composición del terreno y/o propio pilar). Lo más probable es que la fuente de sulfatos sea los terrenos circundantes, partiendo de la base de que los materiales de partida no están contaminados. En el caso de que el ataque se deba a causas externas, es necesario remarcar que se necesita un ambiente con humedad suficiente en los suelos circundantes para que los iones sulfato se muevan, difundan y reaccionen con el hormigón de la estructura.

De nuevo, el análisis de DRX de otro de los pilares de la zona A (pilar E15) no muestra una cantidad de etringita suficiente para deducir un ataque severo por sulfatos a los pilares de forma generalizada.

\section{CONCLUSIONES}

El aspecto visual de los elementos inspeccionados era bueno. Únicamente se encontraron en zonas muy localizadas problemas de corrosión de armaduras.

La inspección por ultrasonidos permitió determinar el estado y calidad del hormigón. Se ha tomado $3000 \mathrm{~m} / \mathrm{s}$ como valor umbral y se han tomado muestras en los elementos que no alcanzaban este valor.

Del análisis del terreno se ha obtenido un alto contenido en yeso, que es fuente conocida de los iones sulfatos que ulteriormente degradan la matriz de cemento de los hormigones.

Del análisis del hormigón de las distintas zonas de la estructura se puede concluir que:

En las zapatas se ha detectado ataque por sulfatos con formación de abundante etringita e incluso taumasita de forma puntual, y son las que peor estado microestructural presentan, debido a que se encuentran en contacto directo con los terrenos ricos en yeso.

Aunque, al igual que las zapatas, los soportes o arranques de pilar están en contacto directo con los terrenos, en general se encuentran en buen estado y no se aprecia productos de degradación expansivos ni fisuración superficial.

En el caso de los pilares, se ha detectado un alto contenido de azufre en zonas bajas por encima de la solera, pero sin observarse daño microestructural. El contenido de sulfatos no es un indicador único para poder diagnosticar la degradación del elemento.

La mayor concentración de cloruros se observa en los pilares de la zona A, disminuyendo a medida que se desciende por el elemento estructural y siendo nula en los terrenos analizados. La fuente de los cloruros es puntual y se debe al uso anterior de la estructura.

Es necesario indicar que, aunque la principal afección a las muestras de hormigón estudiadas ha sido el ataque por sulfatos debido al aporte de los terrenos de relleno antrópico, este no parece ser muy severo ni resulta generalizado a toda la estructura.

Actualmente, está proyectada una reforma en la que (1) se disminuirá el nivel actual de la solera y (2) la sustitución del terreno situado por encima de las zapatas, con lo que se elimina la fuente principal de sulfatos que podría producir un proceso de degradación del hormigón y (3) en cuanto a la degradación por cloruros, no se prevé que existan nuevas fuentes de dichos iones, dado el cambio de uso proyectado.

\section{AGRADECIMIENTOS}

Este estudio se ha realizado en el marco de un Contrato de Investigación suscrito junto a la empresa FERROVIAL AGROMAN S.A. y bajo la tutela y supervisión del Ayuntamiento de Madrid.

\section{REFERENCIAS}

(1) Davenport, A.J., et al., The Structure of the Passive Film That Forms on Iron in Aqueous Environments. Journal of The Electrochemical Society, 2000. 147(6): p. 2162-2173. http://dx.doi.org/10.1149/1.1393502

(2) Maurice, V. and P. Marcus, Progress in corrosion science at atomic and nanometric scales. Progress in Materials Science, 2018. 95: p. 132-171. https://doi.org/10.1016/j.pmatsci.2018.03.001

(3) Maurice, V. and P. Marcus, Passive films at the nanoscale. Electrochimica Acta, 2012. 84: p. 129-138. http://dx.doi. org/10.1016/j.electacta.2012.03.158 
(4) Andrade, C., et al., On-site corrosion rate measurements: $3 D$ simulation and representative values. Materials and Corrosion, 2012. 63(12): p. 1154-1164. http://dx.doi.org/10.1002/maco.201206775

(5) Andrade, C., et al., Analogue circuit of the inductive polarization resistance. Electrochimica Acta, 2011. 56(4): p. 18741880. http://dx.doi.org/10.1016/j.electacta.2010.09.057

(6) Andrade, C., et al. Interpretation of corrosion monitoring from embedded sensors. in SHMII-5 2011 - 5 th International Conference on Structural Health Monitoring of Intelligent Infrastructure. 2011.

(7) Stefanoni, M., U. Angst, and B. Elsener, Corrosion rate of carbon steel in carbonated concrete $-A$ critical review. Cement and Concrete Research, 2018. 103: p. 35-48. https://doi.org/10.1016/j.cemconres.2017.10.007

(8) Angst, U., et al., Critical chloride content in reinforced concrete - A review. Cement and Concrete Research, 2009. 39(12): p. 1122-1138. http://dx.doi.org/10.1016/j.cemconres.2009.08.006

(9) Sanchez, J., et al., Determination of reinforced concrete durability with on-site resistivity measurements. Materials and Structures, 2017. 50(41): p. 1-9. http://dx.doi.org/10.1617/s11527-016-0884-7

(10) Sanchez, J., J. Fullea, and C. Andrade, Fracto-surface mobility mechanism in high-strength steel wires. Engineering Fracture Mechanics, 2017. 186(Supplement C): p. 410-422. https://doi.org/10.1016/j.engfracmech.2017.11.003

(11) Sanchez, J., J. Fullea, and C. Andrade, Corrosion-induced brittle failure in reinforcing steel. Theoretical and Applied Fracture Mechanics, 2017. 92(Supplement C): p. 229-232. https://doi.org/10.1016/j.tafmec.2017.08.006

(12) Garzon, A.J., et al., Modification of four point method to measure the concrete electrical resistivity in presence of reinforcing bars. Cement and Concrete Composites, 2014. 53(o): p. 249-257. http://dx.doi.org/10.1016/j.cemconcomp.2014.07.013

(13) Sanchez-Montero, J., et al., Ten years study of the hygrothermal behaviour of the Prado museum's roof. Informes de la Construcción, 2014. 66(535). https://doi.org/10.3989/ic.12.121

(14) Andrade, C., et al., Evolution of corrosion parameters in a buried pilot nuclear waste container in el Cabril. MRS Online Proceedings Library, 2014. 1665: p. 215-224. https://doi.org/10.1557/opl.2014.648

(15) Torrent, R.J., A non-destructive and rapid method for measuring the permeability of concrete to air. Materiales De Construccion, 1999. 49(254): p. 51-56. https://doi.org/10.3989/mc.1999.v49.i254.450

(16) Andrade, C., et al., Test methods for on-site corrosion rate measurement of steel reinforcement in concrete by means of the polarization resistance method. Materials and Structures, 2004. 37(273): p. 623-643. https://doi.org/10.1007/ BFo2483292

(17) Polder, R., et al., Test methods for on site measurement of resistivity of concrete. Materials and Structures, 2000. 33(10): p. 603-611. https://doi.org/10.1007/BFo2480599.

(18) Andrade, C., et al., On-site measurement of the corrosion rate. Recent Advances in Bridge Engineering - Evaluation, Management and Repair, ed. J.R. Casas, F.W. Klaiber, and A.R. Mari. 1996. 352-366.

(19) Marchand, J., I. Odler, and J.P. Skalny, Sulfate Attack on Concrete. 2004: Taylor \& Francis. https://doi.org/10.4324/97802 03301623

(20) Chinchón-Payá, S., et al., The sulfate attack in concrete by degradation of iron sulfides and the effect of the host rock, in XII DBMC International Conference on Durability of Building Materials and Components. 2011: Porto.

(21) Chinchón-Payá, S., Áridos reactivos en hormigones de presa. Reacción sulfática con formación de thaumasita (PhD Thesis), in Civil Engineering. 2013, University of Alicante: Alicante. http://hdl.handle.net/10045/41565

(22) Yurdakul, E., P.C. Taylor, and H. Ceylan. The Application of X-Ray Fluorescence to Assess Proportions of Fresh Concrete. in Civil, Construction and Environmental Engineering Conference Presentations and Proceedings. 6. 2012.

(23) Bensted, J., Mechanism of thaumasite sulphate attack in cements, mortars and concretes. Zkg International, 2000. 53(12): p. 704-709.

(24) Taylor, H.F.W., Cement chemistry. 1997: Thomas Telford.

(25) BRE_(Building_Research_Establishment). in First International Conference on Thaumasite in Cementitious Materials. 2002. BLDG RES ESTABLISHMENT, Watford, England.

(26) Chinchón-Payá, S., et al., External sulfate attack in dam concretes with thaumasite formation. Materiales de Construcción, 2015. 65(317). https://doi.org/10.3989/mc.2015.10513

(27) Block, C.N., et al., Use of handheld X-ray fluorescence spectrometry units for identification of arsenic in treated wood. Environmental Pollution, 2007. 148(2): p. 627-633. https://doi.org/10.1016/j.envpol.2006.11.013

(28) Kalnicky, D.J. and R. Singhvi, Field portable XRF analysis of environmental samples. Journal of Hazardous Materials, 2001. 83(1): p. 93-122. https://doi.org/10.1016/So304-3894(00)oo330-7

(29) Suarez-Fernandez, G.P., et al., Analysis of major, minor and trace elements in coal by radioisotope $X$-ray fluorescence spectrometry. Fuel, 2001. 8o(2): p. 255-261. https://doi.org/10.1016/So016-2361(oo)ooo88-o

(30) Guidebook on Non-destructive Testing of Concrete Structures. 2002, Vienna: INTERNATIONAL ATOMIC ENERGY AGENCY.

(31) Instrucción de Hormigón Estructural. EHE-o8 comentada. 2010.

(32) Meyer, A., Normas para la determinación de las resistencias del cemento. Materiales de Construcción, 1964. 14(115): p. 19-43. https://doi.org/10.3989/mc.1964.v14.i115.1779 